\title{
Elimination of preventable blindness: can success in Nepal be replicated in Africa?
}

This article was published in the following Dove Press journal: Innovation and Entrepreneurship in Health

\section{Neda Nikpoor ${ }^{1,2}$ \\ Eric D Hansen ${ }^{2,3}$ \\ Matthew S Oliva ${ }^{2,4}$ \\ Geoffrey Tabin ${ }^{1,2}$ \\ Sanduk Ruit ${ }^{2,5}$}

'Byers Eye Institute, Stanford University, Palo Alto, CA, USA; ${ }^{2}$ Himalayan Cataract Project, Waterbury, VT, USA; ${ }^{3}$ Moran Eye Center, University of Utah, Salt Lake City, UT, USA; ${ }^{4}$ Casey Eye Institute, Oregon Health and Science University, Portland, OR, USA; ${ }^{5}$ Tilganga Institute of Ophthalmology, Kathmandu, Nepal

Correspondence: Matthew S Oliva

Himalayan Cataract Project, PO Box 55, Waterbury, VT 05676, USA

Tel +l 8882878530

Email moliva@cureblindness.org

\begin{abstract}
There are an estimated 36 million people who are blind worldwide: $\sim 1$ in every 200 people. A third of these are blind from cataracts and could have perfect vision restored with a simple operation costing less than US\$25. Ninety percent of the blind and visually impaired live in the developing world, with particular concentrations seen in South Asia and sub-Saharan Africa (SSA). Innovations in surgical techniques, delivery systems, and material production, developed first in Nepal and India, have provided a viable blueprint to address treatable blindness in the developing world. In fact, Nepal was the first developing country to reverse its trajectory of blindness. This was largely possible because of a collective effort by many international nongovernmental organizations to support Sanduk Ruit and his colleagues as they developed a sustainable eye care-delivery model anchored at Tilganga Institute of Ophthalmology. This model is similar to that of LV Prasad Eye Institute and Aravind Eye Hospital in India, which is also being successfully utilized to combat the enormous burden of needless blindness in Africa. Over the last decade, there have been early successes in transferring the Nepali model of care to Ethiopia and Ghana, and there have been other models successfully implemented across Africa as well. Though there has been significant progress in restoring sight in SSA, the prevalence of avoidable blindness in Africa is higher than any other continent, with about 4.8 million people living blind.
\end{abstract}

Keywords: blindness, cataract,visual impairment, Nepal, Africa, cataract surgery

\section{Introduction}

There are an estimated 36 million people who are blind worldwide: $\sim 1$ in every 200 people. ${ }^{1,2}$ A third of these could have perfect vision restored with a simple operation costing less than US\$25. Data from the 2010 Global Burden of Disease study estimated that there were 32.4 million people blind globally, yet these numbers may underestimate the magnitude of global blindness. World Health Organization (WHO) data placed the number of globally blind closer to 39 million in $2010,{ }^{3}$ and all data sources agree that the burden of global blindness continues to increase. ${ }^{1-4} \mathrm{~A}$ growing and aging population magnifies the blindness problem and heightens the urgency of implementing a viable solution capable of reaching a global scale.

The effects of blindness are not distributed equally across populations. Ninety percent of the blind and visually impaired live in the developing world, with particular concentrations seen in South Asia and sub-Saharan Africa (SSA). Blindness also exerts its effects disproportionately across social strata within a country or region. Blinding conditions most commonly affect older people (age $\geq 50$ years) in these societies, 
necessitating children and/or grandchildren to remain at home to care for the blind, thereby taking them out of school or out of the labor force. In some countries within these regions, the prevalence of blindness in older adults exceeds $5 \% .{ }^{1}$ In fact, almost a quarter of the population over 50 years old is visually impaired in many developing countries. ${ }^{1}$ What emerges from such sobering statistics is the inexorable link between blindness and poverty, each perpetuating and magnifying the other.

However, $80 \%$ of global blindness could be cured or could have been prevented. ${ }^{1,3,4}$ This key statistic offers possibility and opportunity while highlighting a collective failure of the global community, bringing into focus the moral imperative facing this generation. Among the 36 million blind, $\sim 12.4$ million are needlessly blind due to treatable cataracts. ${ }^{1}$ Cataracts, or opacification of the eye's natural lens, represent the leading cause of blindness worldwide. This is despite cataracts being totally curable with an operation that takes 5 minutes and has a material cost of about US\$25.

Innovations in surgical techniques, delivery systems, and material production, developed first in Nepal and India, have provided a viable blueprint to address treatable blindness in the developing world. However, the lingering question for international eye-care nongovernmental organizations (NGOs) and policy makers remains: Can the successes in South Asia can be effectively transferred to the unique realities of SSA?

\section{Cataract blindness}

Cataracts occur when the eye's natural lens undergoes changes resulting in progressive opacification of the lens and decreased vision. These changes are primarily a process of aging; however, certain medical conditions, medications, and environmental exposure can hasten cataract progression and thus visual deterioration. Besides age, known risk factors for cataract development include significant ultraviolet-light exposure, smoking, poor nutrition, exposure to household smoke, diabetes, and eye trauma. The treatment for cataracts is surgical extraction with implantation of an intraocular lens.

This year in the USA, about 3.6 million people will undergo cataract surgery (CS). ${ }^{5}$ At the time of surgery, the majority of these patients will have vision that still allows them to drive. In fact, CS is the most common surgery performed in the USA, and over 20 million CSs are performed worldwide each year. ${ }^{5}$ Continual innovation within ophthalmology has made the surgery routine, increased efficiency, improved outcomes, decreased complications, and speed recovery times. The prevailing technology used for CS in the Western world, phacoemulsification, uses ultrasound technology to break apart the cataractous, opacified lens in the eye, often through an incision only $2.2 \mathrm{~mm}$ wide, and then removes the emulsified pieces so that an artificial lens can be inserted into the eye. The entire procedure can take as little as 5 minutes, start to finish.

Despite such advances, more than 12 million people worldwide are blind in both eyes due to cataracts. ${ }^{1}$ These people, bilaterally blind by WHO standards, are unable to complete the tasks of daily living as a result of their vision. Such barriers as cost and access to care hinder people in low- and middle-income countries from reaching known, cost-effective solutions to cataracts as well as other treatable blinding eye diseases.

As the leading cause of global blindness and the foundation of surgical ophthalmic care, cataract has been and will continue to be at the heart of meaningful efforts to eradicate the world of treatable blindness. If the story of avoidable blindness is to be rewritten, sight-restoring CS will be at its forefront. Fortunately, a new narrative has already been told in Nepal, one of the most poverty-stricken and austere environments on Earth.

\section{Lessons from Nepal Background}

Nepal is one of the only countries in the developing world to reverse its rate of blindness. The manifold lessons gained from Nepal's transformation are informing coordinated efforts to achieve the same in other regions, especially SSA. In 1981, a national blindness survey conducted in Nepal found the prevalence of blindness to be $0.84 \% ; 72 \%$ of blindness in Nepal was caused by cataract. ${ }^{6}$ At the time, the national blindness survey was published, phacoemulsification was the emerging technique for CS in developed countries. However, phacoemulsification surgery posed significant challenges in the developing world, due to the high cost of necessary medical equipment and consumables, in addition to a steep learning curve for the surgeon. In the early 1990s, the least expensive intraocular lens implant on the world market was priced at US\$200, more than the average annual income of a Nepali citizen and thus unattainable for all but the wealthiest Nepalis.

The landscape of eye care in Nepal at this time was further complicated by a paucity of trained ophthalmologists and few suitably trained ophthalmic nurses and ophthalmic assistants. Nepalese surgeons were not performing modern microsurgical cataract operations with routine intraocular lens implantation, and training programs or formal eye-care 
systems were nonexistent. In addition, governmental input for eye-care delivery was minimal, as eye health lacked prioritization amid an anemic economy and a constrained budget. Given these realities, the total number of CSs performed in Nepal hovered around 1,500 cases per year. Even then, the majority of surgery was done only in the Kathmandu valley, neglecting the majority of the blind who lived in rural Nepal. As a result, Nepal had a backlog of 300,000 people blind from cataracts in 1990, with 60,000 new people going blind every year. ${ }^{7}$ Considering the lack of trained clinicians and the cost involved, the prospect of eliminating blindness seemed insurmountable.

Facing these obstacles, ophthalmologist Sanduk Ruit returned to his native Nepal in the early 1990s, having trained in India, the Netherlands, and Australia. He set out to improve eye care in Nepal and prove that it was possible to provide modern, high-quality services to all patients, irrespective of their ability to pay. Ruit's vision galvanized the support of many international NGOs, including the Fred Hollows Foundation, the Himalayan Cataract Project (HCP), SEVA Foundation, Christian Blind Mission, and others. The international community formed a united front to address the problem of cataract blindness in Nepal. The Nepal Eye Program, a not-for-profit, community-based NGO, was officially launched in 1992 to support the prevention and control of blindness in Nepal. Ruit and his colleagues opened the Tilganga Institute of Ophthalmology (TIO), as the implementing body of the Nepal Eye Program in 1994, in Kathmandu. TIO was founded to serve as a center of excellence for delivery of tertiary care and training at all levels of eye-care delivery. As a not-for-profit model, the tenets of TIO focused on training, infrastructure development, and delivery of $\mathrm{CS}$ to the maximum number of patients at the lowest possible cost. TIO began close collaboration with other emerging hospitals in Nepal, such as Lumbini, Lahan, and Biratnagar.

Limited by cost and number of ophthalmologists, TIO developed an innovative delivery system for outreach that provided low-cost, high-quality care by employing a team approach that maximized each member's performance and utilized local lay volunteers in remote areas. This system empowered a small team of one to two ophthalmologists and a cadre of ophthalmic assistants working with local partners to provide hundreds of CSs in a remote setting within just a few days.

With Fred Hollows Foundation support, TIO established the first intraocular lens manufacturing facility in Nepal, cutting the unit cost of an intraocular lens from near US\$200 to $<\mathrm{US} \$ 4$. During this time, Ruit et al were also innovating a sutureless, extracapsular manual small-incision CS (MSICS) technique that would eliminate the need for costly, complex instrumentation and expensive consumables. This innovation on manual CS techniques represents one of the most significant steps forward for global ophthalmology. ${ }^{8}$

In trained hands with a team approach, MSICS can consistently be completed in $<5$ minutes with low rates of complications, while allowing rapid turnover of both patients and instrumentation. Ruit et al had the MSICS technique published in 2000 in the journal Clinical and Experimental Ophthalmology. ${ }^{8}$ By 2007, numerous independent studies had proven the technique carried equal visual outcomes with similar rates of complications to phacoemulsification, the prevailing technique utilized in the Western world. ${ }^{9,10}$ In 2007, a prospective randomized trial was conducted to compare MSICS to phacoemulsification on typical Nepalese cataracts in the hands of expert surgeons. ${ }^{10}$ The results showed equivalent visual outcomes, yet MSICS was faster, cheaper, and easier for the advanced cataracts that are often encountered in the developing world. Not only did this technique reduce the material cost of CS to $\sim \mathrm{US} \$ 25$ but it also supported an infrastructure in which a single surgeon could routinely perform over 100 sight-restoring CSs per day. A cost-recovery model was initiated in which patients paying for modern CS in areas surrounding the Kathmandu valley helped subsidize free or reduced-cost care for others, specifically in rural areas. A large number of outreach sites were quickly developed, and with time many of these sites have developed into full-time primary eye-care centers staffed by ophthalmic assistants.

TIO's network of community eye centers in Nepal was created so that patients in rural areas would not be dependent solely on outreach campaigns for easily treatable conditions. Patients who cannot be treated at small clinics are referred to TIO for treatment, and efforts are under way to implement a telemedicine system to optimize the referral system. The TIO system maximizes efficiency and utilization of personnel by delegating tasks strictly according to level of training and avoiding task redundancy. With its innovative cost-recovery model and efficient utilization of resources, TIO created a model of sustainability that could provide financial incentives to retain highly talented surgeons, nurses, and staff. Throughout Nepal, other ophthalmologists and hospitals adopted this model of cost recovery, thereby creating an environment in which the quality of care delivered was elevated throughout the country and profits redirected to sustain good salaries for doctors, nurses, and hospital workers. Once this model was 
in place, cataract-blindness rates were reversed, and there was a parallel focus on development of subspecialty and preventive ophthalmology. Today in Nepal, all subspecialties are practiced and taught and other causes of blindness prevented with primary eye care.

\section{Training and infrastructure}

With the support of committed NGOs, which made key investments in constructing a training center, acquiring basic equipment needs, such as operating microscopes and instruments and supporting the intraocular lens factory, TIO grew quickly in size and scope. In the last 20 years, TIO has grown from a small facility with 12 employees to a large, state-of-the-art institution with over 400 employees, which has served more than 4.6 million patients. In addition to its $12,356 \mathrm{~m}^{2}$ facility, TIO operates 15 satellites, including one community eye hospital in Hetauda and 14 community eye centers throughout rural Nepal. Each year, the TIO network trains hundreds of ophthalmic personnel from 43 different countries.

TIO now has a world-class residency training program that draws the top young medical students in Nepal. Initially, ophthalmologists underwent enhanced CS training in Nepal but were only able to pursue subspecialty fellowships abroad. Today, all ophthalmic subspecialties are taught and practiced at TIO, and dozens of ophthalmologists from other countries in Asia and SSA receive advanced subspecialty training in Kathmandu.

TIO's training program also offers rigorous training for mid-level ophthalmic personnel, including a 3-year ophthalmic assistant training program, a 2-year optometrist program, and several short-term exposure courses in various topics, such as ophthalmic equipment maintenance and outreach management. These skilled nurses and technicians function as mid-level care providers and power a large network of primary eye-care facilities across the country.

\section{Compassionate capitalism and innovation} In Nepal and India, such organizations as TIO, Aravind Eye Institute, and the LV Prasad Eye Institute employ a refined and affordable eye care-delivery model, whereby a high percentage of patients are able to access free or subsidized care. Aravind Eye Institute pioneered this model around the world, applying business principles to eye care and focusing on serving those who would otherwise have no access to services. ${ }^{11}$ The tenets of this strategy include:

- Specialization in high-volume eye care, with highly trained physicians, nurses, and administrative staff, using a standardized system engineered for high-volume production
- High volume and efficiency that enable the unit cost to fall dramatically compared to traditional low-volume settings

- Combination of operational efficiency with high-quality service delivers exceptional outcomes, which attract and retain a high volume of patients (from high to low incomes)

- Using profit and production capacity to serve low-income clients $^{12}$

This model underscores the critical role of training, the value of high-volume care, and a commitment to excellent outcomes. Integral to this is a focus on low-cost, portable equipment development to enable delivery in remote settings. With incentives to promote excellence, Aravind was able to provide high-quality, high-volume care at a low cost and scale up rapidly from a 20-bed hospital in 1976 to an institute with 250 beds, state-of-the-art equipment, and all major ophthalmic subspecialties by 1981. In part, the success of Aravind is due to the assembly-line model. In this model, the services offered are defined and the labor needed broken into constituent parts. These labor tasks are then fine-tuned and reassembled until peak efficiency is achieved, all while maintaining consistent high quality, low cost, high customer/ patient satisfaction, and continued growth of profits. Unlike many other surgical procedures, CS is the same surgery performed repetitively with little variation and lends itself well to the assembly line model. As a result of efficient cost recovery, Aravind is able to provide free service to two-thirds of their patients. For each US\$1 spent, US\$1.60 is earned. An excellent electronic medical record system also facilitates administrators to examine and optimize patient flow.

TIO adopted the model of compassionate capitalism developed by Aravind and adapted it to Nepal's socioeconomic and cultural landscape. Financial counselors, refractive laser surgery and after-hour "private" clinics that provide identical patient care with decreased wait times are some of the unique adaptations that drove this model's success in Nepal. Such innovations maximized the amount of free or subsidized eye care that could be delivered in the Kathmandu valley and rural Nepal.

\section{Successes in Nepal}

With its efficiencies and incumbent techniques, the highquality eye-care system developed in Nepal increased the CS rate (CSR) of Nepal by $1,000 \%$ over the last three decades, to an estimated $4,364 .{ }^{4}$ The CSR, a quantifiable measure of CS-service delivery, is the number of CSs per million population per year. Typically, in economically developed countries, the general CSR ranges from 4,000 to 10,000 , 
and cataract blindness is very rare. ${ }^{4}$ In contrast, in many African countries, the CSR is reported to be $<500 .{ }^{4}$ Moreover, the quality of CS performed in Nepal has elevated to a level equivalent to anywhere in the world, including medical systems in developed countries. In 2015, nearly 350,000 high-quality CSs were performed in Nepal. ${ }^{13}$ These transformations paralleled a similar rise in access to primary eye-care services and delivery of subspecialty care. Due to these efforts and advances, the prevalence of blindness in Nepal dropped from $0.84 \%^{6}$ in 1985 to $0.35 \%^{7}$ in $2012-$ a $58 \%$ decrease. In 2004, for the first time in the nation's history, there were more CSs performed in Nepal than new cases of cataracts reported. Today, Nepal serves as a shining example of a country that has reversed its rate of blindness. ${ }^{6,7}$

The innovations in Nepal - the sutureless CS technique, the intraocular lens-manufacturing facility in Nepal, and the outreach system - supported by committed NGOs, proved that delivering care to the poorest of the poor and developing state-of-the art eye care in a "compassionate capitalism" model is feasible and sustainable. It has also shown the ability to coordinate efforts throughout the country with all eye-care workers and the NGOs. Broad applicability of the CS-outreach delivery model has been demonstrated through similar successes in neighboring Bhutan, areas of India, and now SSA. The transformation of Nepal's eye care occurred through a crucible of political obstacles and natural disasters: the prolonged Maoist revolution and the devastating earthquakes in 2015. With a steadfast focus on training and achieving quality outcomes in the setting of limited human and financial resources, Nepal has shown that it is possible to reach the unreachable patient and provide successful surgical outcomes anywhere.

\section{The African landscape}

Africa is home to $\sim 17 \%$ of the world's population, has $24 \%$ of the global disease burden, yet contains only $3 \%$ of the healthcare workforce. Challenges that have retarded progresses in healthcare development in this continent are well documented, yet effective solutions often remain elusive and poorly elucidated. ${ }^{14}$ Within eye care, which has traditionally received little priority, these realities are especially stark. Though there has been significant progress in reducing vision loss in SSA, the prevalence of avoidable blindness in Africa is higher than any other continent, with about 4.8 million people living blind. ${ }^{1,4}$ Admittedly, the region is large and diverse, and there are many successful eye-care programs in Africa; however, data show that as a whole, the SSA region has seen the least progress since 1990 in combating cataract blindness. ${ }^{1,2,4}$ As population growth in SSA continues to outpace other regions of the world, these challenges are poised only to grow larger and more complex.

There are unique challenges in the African landscape that make the simple transfer of the TIO or Aravind eye care-delivery model difficult. Africa is an exceedingly diverse continent, and generalizations both between and even within countries carry the risk of gross oversimplification. Despite the challenges, there have been many success stories of national eye-care development described in the literature. ${ }^{15}$ Acknowledging the broad range and diversity of SSA and the limited scope of this paper, we focus our discussion on specific examples relevant to the implementation of the Nepali model of eye-care delivery. Specifically, we use Ethiopia and Ghana as two examples to attempt to illustrate some of the successes and challenges encountered in efforts to implement the Nepali model in SSA.

\section{Epidemiology}

In the past, much focus was directed to infectious eye diseases in SSA, such as trachoma, onchocerciasis, measles-related blindness, and vitamin A deficiency. Prevention of these conditions through successful public health programs resulted in decreased prevalence of these causes of blindness, and they now contribute much less to the overall burden of blindness in SSA. ${ }^{15-17}$ Today, however, the most common causes of vision loss are cataract, refractive error, and posteriorsegment diseases (mainly glaucoma, diabetic retinopathy, and age-related macular degeneration). While prevention of infectious diseases requires minimal specialized knowledge of ophthalmology, diagnosis and treatment of posterior segment diseases demands highly specialized tools and training.

Cataract blindness in SSA is responsible for 35\%-45\% of blindness, depending on the region, according to recently published (2015) data from the Global Vision Database. ${ }^{1}$ The remaining blindness is due to uncorrected refractive error $(12 \%-13 \%)$, glaucoma $(12 \%-15 \%)$, trachoma $(0-7 \%)$, age-related macular degeneration $(3 \%-12 \%)$, corneal opacity $(3 \%-4 \%)$, diabetic retinopathy $(0-1.5 \%)$, and other causes (17\%-19\%), with some variation among central, east, west, and southern SSA. ${ }^{1}$ Comparatively, in South Asia, the prevalence of cataract blindness is $36.58 \%$, whereas other cases are accounted for by uncorrected refractive error $(36.43 \%)$, glaucoma $(5.81 \%)$, trachoma $(0.04 \%)$, age-related macular degeneration $(2.44 \%)$, corneal opacity $(2.43 \%)$, diabetic retinopathy $(0.16 \%)$, and others $(16.1 \%) .{ }^{1}$ The higher prevalence of noncataract, nonrefractive error causes of blindness further hinders the eradication of avoidable blindness in SSA. 
Glaucoma and macular degeneration, both chronic conditions that are difficult to treat in even a high-resource setting, are more prevalent in SSA, where resources are limited. Furthermore, patients are generally willing to pay for curative services like CS and spectacles, but may be unwilling to pay for or endure preventive treatment for such conditions as glaucoma, a condition for which surgery can be painful and does not improve vision.

\section{Barriers to implementing care in SSA}

The unique geographic, socioeconomic, and political realities of many African countries, especially those in SSA, have hindered the progress of eye care-system development. Lack of infrastructure and roads, anemic supply chains, political corruption and unrest, and limited financial resources in many countries in SSA have plagued efforts by NGOs and health ministries to develop viable national eye-care plans.

The most daunting barrier is the paucity of personnel dedicated to eye-care health. Properly trained eye-care personnel producing high-quality and high-volume CS is a primary need, in order to halt and eventually reduce the rising backlog of cataract blindness in Africa. ${ }^{18}$ Highly trained doctors and eye-care teams are inextricably linked to the quality of services rendered. Excellent high-quality outcomes in turn generate patient demand for these services, regardless of the environment. ${ }^{19}$ A happy patient returning from a successful CS outreach event generates the best marketing and incentive for others to pursue surgery and access care. Institutionalizing quality is dependent upon continuous training and skill transfer to empower providers at all levels, from technicians to subspecialty-trained ophthalmologists.

SSA faces enormous challenges in increasing the requisite personnel. The number of ophthalmologists per capita is about 2.9 per 1 million people, ${ }^{20}$ among the lowest in the world. The situation is exacerbated, as almost two-thirds of these are concentrated in urban centers, whereas the majority of the population live in rural areas. In addition, the productivity (CSs per surgeon per year) of African surgeons is only about 178 per surgeon per year, once again the lowest in the world. There are very few ophthalmologists or nonophthalmologist cataract surgeons who are suitably trained and experienced in providing simple, inexpensive, high-quality CS services, particularly MSICS. Anecdotally, this is partly because residency training programs do not adequately prepare surgeons with enough hands-on surgical experience. For example, in Ethiopia, resident physicians are required to perform $100 \mathrm{CSs}$ prior to graduation. However, most residents have not reached this threshold by the time of their scheduled graduation, and additionally the majority of their surgeries are performed without a senior surgeon supervising them at the microscope.

In response to the perceived shortfall in ophthalmologists, many SSA countries adopted a task-shifting strategy in which nonphysician cataract surgeons (NPCSs) are trained. Such countries as Tanzania, Kenya, Malawi, and the Gambia have embraced this approach. NPCS training usually occurs in formal 1- to 2-year programs supported by NGOs and ministries of health. However, this approach also has its limitations. Data on NPCSs in eastern Africa indicate that the number of CSs per year per surgeon has not reached its potential. For instance, one study in Ethiopia found that the productivity of NPCSs (CSs per surgeon per year) was 280 compared to 682 for ophthalmologists in the same area. ${ }^{21}$

Similarly, task shifting of trichiasis surgery for trachoma to general healthcare workers began in 2007 , but the median number of surgeries per surgeon was again lower than anticipated. Inadequate surgical output is likely due to the lack of support for outreach, a dearth of supplies, including instruments and consumables, and inadequate supervision. For instance, a 2011 study in Ethiopia found only about 3\% of surgeons had all the essential items to perform trichiasis surgery. ${ }^{22}$ As a result of these findings, most trachoma-endemic countries have shifted provision of trichiasis surgery back to dedicated eye-care personnel, generally ophthalmic nurses or ophthalmic clinical officers.

Task shifting has shown limited efficacy as a solution to the shortage of personnel for eye health in SSA. Investments to address the productivity of existing surgeons would likely improve services without the additional cost of training new personnel, whereas training more people who are not then empowered to work productively is poor use of already strained resources. Additionally, efforts to improve the quality of care delivered and increase patient volume are also hindered by insufficient surgical supplies, inoperative wet labs, and governmental regulations that hinder sustainable reimbursement strategies. Additionally, eye-care providers who are well trained often lack the ancillary support to maintain the equipment necessary to perform highly technical and specialized tasks. Efforts have been made to train technicians and biomedical engineers, but this human infrastructure and the requisite supply chains are slow to develop, so availability and maintenance of equipment remains a significant challenge. In addition to personnel, financial resources are also limited. Ministries of health often focus investments on malaria, HIV/ AIDS, and maternal and child health in lieu of prioritizing eye 
care. This occurs despite a well-documented high-economic return on investment and societal impact for eye care in the developing world. ${ }^{23}$ In 2001, African governments committed to increase investments in health to $15 \%$ of national budgets by 2015 , yet by 2011 only six had achieved this target. ${ }^{24}$

Several factors that spurred success in Nepal and India could hinder transference in SSA if they remain uncorrected. Quality outcomes are paramount for successfully implementing and sustaining eye-care programs. High-quality CS is readily understood by patients as nearly perfect vision with minimal discomfort. Complicated surgery can result in pain or worse vision. Lewallen and Thulasiraj reviewed recent population-based surveys and found rates of blindness (vision $6 / 60$ or worse) after CS in Africa as high as $23 \%-58 \% .{ }^{25}$ Poor vision following $\mathrm{CS}$ can result from poor surgical technique, incorrect lens selection, or failure to screen for concomitant eye disease. Unfortunately, poor vision following surgery, irrespective of the reason, undoubtedly decreases demand and lowers patient volume. In addition to maximizing overall patient volume, a key component of sustainable success is capturing the middle and upper classes within a country that are willing to pay for additional services. For example, at Aravind, this might include patients seeking newer technology and premium intraocular lenses, finer food, private rooms, or other amenities during their surgical experience. While this model can work in urban Africa, difficulty arises in predominantly rural populations, where anecdotal evidence suggests that almost all patients in rural SSA opt for free care. ${ }^{25}$ This component of the cost-recovery model remains underdeveloped in SSA.

TIO and Aravind employ a team approach, with highly trained staff supporting the surgeon. This facilitates highvolume surgery and comprehensive care delivery. As previously discussed, eye surgeons in SSA are often unsupported or supported with insufficient and inadequately trained personnel, and surgeons often waste valuable time on managerial or equipment maintenance tasks, thereby decreasing productivity. ${ }^{25}$ India also has a high population density, which makes gathering large numbers of patients to support a high-volume, low-cost system easier than in SSA, where underdeveloped roads and a widely distributed rural population pose greater challenges. Understanding these barriers is necessary to direct future investments and best allocate resources. With well-focused efforts and sustained support to build capacity in properly aligned local partners, such barriers can be overcome to realize growth in the African landscape.

\section{Successes in SSA}

Even amid such limitations, bright spots are emerging on the continent, and there have been examples of successful transference of the South Asian models to SSA by various groups. Premier successful eye institutes from India have built several eye centers in Africa. At the invitation of Liberia's president, the LV Prasad Eye Institute has opened an eye hospital in Monrovia based on the same tenets of care as their flagship institution in Hyderabad, India. Similarly, the leaders of the Aravind Eye Hospital have set out to replicate their successful model through collaborations in Ethiopia, Kenya, Zambia, and Nigeria. ${ }^{26}$ In partnership with the Conrad Hilton Foundation and the Dana Center for Preventive Ophthalmology, the Aravind model is being scrutinized and refined in diverse African settings. In conjunction with numerous partners, the International Council of Ophthalmology has founded a comprehensive eye-hospital model in Cameroon to replicate a center of excellence with service and training components in central Africa. ${ }^{27}$ The Kilimanjaro Centre for Community Ophthalmology has been working since 2001 in Tanzania to implement and evaluate comprehensive community ophthalmology services and training in east Africa and beyond. ${ }^{28}$ Public health in primary-care ophthalmology has seen great improvements in the Gambia, as well as marked progress in reducing onchocerciasis and trachomatous blindness from multiple organizations. ${ }^{15}$ There have also been efforts from several top-quality Indian organizations, such as Dr Agarwal's Eye Hospital branches in ten countries in Africa. ${ }^{29}$ There are many other groups working across Africa to eradicate needless blindness and improve eye-care delivery. Finally, HCP initiated work in SSA in 2006 in a partnership with the Earth Institute's Millennium Villages Project (MVP), a group of 14 research villages that helped inform the development of the United Nations Millennium Development Goals. This was the beginning of their efforts to lay the foundation for transference of the Nepal model. All of these groups' efforts have resulted in nurturing a nascent south-south connection, with meaningful and long-term relationships between South Asia and SSA.

Within the MVP initiative, HCP screened over 20,000 people and provided CS to over 800 patients in millennium villages in Ghana, Ethiopia, Uganda, Rwanda, Kenya, Nigeria, Tanzania, and Malawi. During this work, HCP established strong relationships with the local eye-care providers and offered skills, tools, structures, and technical support to begin building or bolstering their national eye-care systems. Projects in Rwanda, Ghana, and Ethiopia were extended in 
large part due to the interest and initiative of the local eye-care providers, with whom HCP has maintained lasting relationships. The most successful eye-care systems come from the will and determination of local people, not from outside. As such, successful eye-care NGOs must view their role as partner-trainer-supporter of a human initiative to build care for a country's people.

Among one of the brightest examples of success in SSA is Rwanda, where supported key leaders like ophthalmologists John Nkurikiye and Ciku Mathengue have been successful in their efforts to integrate eye-care services into the country's community-based health insurance and have broadened surgical coverage with the same focus on quality outcomes and training that guided TIO and Ruit's work in Nepal. Since 2006, Rwanda has increased its CS coverage for blind patients from $47 \%$ to $68 \%$. In the same time frame, the CSR in Rwanda has increased by $33 \%$. $^{30,31}$

Since 2006, HCP has focused the majority of its financial resources to efforts in Ethiopia and Ghana, due to strong engagement and self-determination of local partners. Additionally, these countries offered the chance to demonstrate that the Nepalese model of blindness reduction and eye-care improvement can be successful in countries with varied geography, political systems, and national healthcare systems, eg, a private health system (Nepal), a public system (Ethiopia), and a hybrid public-private system (Ghana).

Efforts to scale and replicate the eye-care service-delivery model that was proven effective in Nepal into Ghana and Ethiopia are well under way through investment in direct clinical care, training opportunities, and infrastructure improvements in each country. Advocacy, collaboration with stakeholders and policy makers, and coalition building complete the model and project its impact on a macro-, system-oriented level. Such a multifaceted approach is requisite for developing an effective national eye-health system that will succeed in blindness elimination and low-vision improvement.

This strategy, utilized in Nepal and now transferred to Africa, aligns with global strategies to address avoidable blindness. The WHO's 2006 adoption of a prevention of avoidable blindness and visual impairment resolution ${ }^{32}$ helped foster the global mandate to address blindness. It advocates three core approaches: training the workforce, expanding infrastructure and technology, and delivering clinical services to control/treat disease. Although distinct components, their interdependence is evident: clinical services require an adequately trained, functional workforce with supportive infrastructure and technology. The prevention and elimination of avoidable visual impairment can only be achieved if effective, efficient, comprehensive eye-healthcare services are integrated into well-managed, well-monitored national health systems.

\section{Transferring the model}

Replication of Nepal's success in Ethiopia and Ghana has reached its adolescence. Though no two countries can wholly represent the entire continent of Africa, the challenges in these countries mirror the broader challenges facing SSA: limited and undertrained personnel, underdeveloped infrastructure and supply chains, and poor patient confidence and awareness.

Particularly in Ghana, low surgical output by existing ophthalmologists is closely tied to insufficient surgical experience in residency training programs, lack of incentives to provide CS, and shortage of supplies. In addition, there are only 340 allied ophthalmic personnel for the entire country, which further limits ophthalmologist productivity. Inadequate funding has also hindered Ghanaian eye-care development. ${ }^{30}$ Ghana established its National Health Insurance Scheme based on district-wide mutual health insurance schemes in 2003 , and by 2008 reportedly $61 \%$ of the population was covered. ${ }^{33,34}$ However, though the list of benefits grew to encompass most eye surgeries and diagnostics, increased access and raised public expectations were not met by an increase in funding for these programs. Indeed, many ophthalmologists reported delayed or nonexistent reimbursements for services reportedly covered by the scheme.

Despite such limitations, Ghana has seen success with the Komfo Anokye Teaching Hospital (KATH) in Kumasi, whose partnership with HCP began in 2006. Several US Agency for International Development grants financed the construction and equipping of a 2,500 $\mathrm{sm}^{2}$ modern eye center separate from the main hospital with three operating rooms and ample clinical space dedicated for ophthalmology. The majority of KATH's eye-care staff have received specialized training at TIO, the USA, India, and/or their home institution during hands-on training workshops. The CSR in the Ashanti region has doubled, and $\mathrm{KATH}$ has increased its surgical output tenfold since $2006 .{ }^{4}$ Most recently, HCP has been supporting the establishment of national outreach guidelines to be adopted by the health ministry that slowly transition the financial responsibility of outreach to the government, an effort that would make continued success in Ghana more sustainable and less dependent on foreign aid and NGOs.

Ethiopia also shares the system-wide hurdles that hinder large-scale eye-care delivery in SSA. The limited number of ophthalmologists and lack of incentives are significant 
barriers. Specifically, there are 150 ophthalmologists for a country of 102 million, with only two-thirds currently performing CS. ${ }^{4}$ Over half of these ophthalmologists are then concentrated in the capital, while the majority of poor, blind patients live in rural areas with limited transport infrastructure. The majority of ophthalmologists work in the public system, where remuneration is low compared to other surgical subspecialties. And though private-practice opportunities that generate additional income are improving, patient volume and reimbursement remain generally depressed. There are few ophthalmic nurse-training programs and insufficient financial incentives present to make ophthalmic nursing highly desirable on a wide-scale basis. In fact, though Ethiopia has a population $400 \%$ that of Nepal, it houses 33\% less supporting allied ophthalmic personnel.

Since the MVP, there has been steady progress in eyecare development by local leaders, backed by committed NGOs. The high-volume model that was perfected in Nepal is now functioning well in the Ethiopian context. In 2016, HCP supported 29 outreach surgical campaigns fashioned after the Nepal model, resulting in 12,533 surgeries or $20 \%$ of the estimated total surgeries performed in Ethiopia that year. HCP also supported 63 specialized training opportunities for ophthalmic personnel, ranging from 2-day planning workshops to 12-month subspecialty fellowships. ${ }^{4}$

Successful partnerships in Ghana and Ethiopia can be attributed to the adaptation of the Nepalese model which drove TIO's success. Identification of properly aligned local partners, whether institutions or individuals, is critical to the replicability and sustainability of the systems being implemented. There must be local empowerment through skills transfer and infrastructure investment, focus on quality, and commitment to adequate follow-up, in order to reach the same tipping point Nepal reached in its efforts to eradicate needless blindness.

Given the insufficient number of properly trained ophthalmic providers in SSA, training initiatives are a critical component in success. Principles taken directly from Nepal's playbook have been integral in shaping the trajectory of work in Africa. Training initiatives encompass all levels of providers, from technicians to subspecialty physicians, and once trained, each individual's potential is maximized within a system that also seeks to maximize efficiency.

Impartation of the high-volume system has occurred through varied, complementary means, and a powerful south-south training connection. Lead nurses, promising allied-health personnel, and ambitious doctors looking to increase their skills and reach are routinely sent to TIO in
Kathmandu or LV Prasad or Aravind in India for formal training courses. Exposure to highly coordinated, efficient, and replicable outreach systems combined with hands-on training impart knowledge and skills that can be immediately and directly applied locally upon their return home.

Nepalese trainers also travel to Africa to follow up with former trainees, perform in-country skills transfer, including "train the trainer" courses, to facilitate the replication of the Nepalese model in the trainee's unique environment. High-volume surgical outreaches are an important piece of the transference puzzle. For example, all HCP-supported outreach campaigns in Ghana and Ethiopia include one surgical station dedicated to one or two ophthalmology residents to ensure they meet the program requirements while improving surgical skills and learning from senior surgeons. The residents are supervised by either a local trainer or an HCP surgeon. These high-volume surgical campaigns also provide skills transfer for nurses and technicians. US trainers, Nepalese trainers, and African trainers all function within this system, where high-volume output parallels high-volume training. The results of this approach are evident in Ethiopia, where local teams now have surgical output reaching 100-200 surgeries per day.

\section{Looking to the future}

Transference of the high-volume service-delivery model pioneered in Nepal is only a piece of the larger replication puzzle. Institutionalizing quality, building capacity in local centers of excellence, inspiring government investment, strengthening primary eye care, and encouraging buy-in by the larger ophthalmic community within a country are equally important. The leadership and prominence of Ruit in coordination with convergent NGO investment in eye care cannot be ignored as unique factors precipitating the transformation seen in Nepal. What will similar convergent forces that stimulate change in African countries be? Who will the leaders that galvanize change be? What will the source of revenue streams and supply chains necessary for change be? Answers to such questions are beginning to emerge.

\section{The importance of quality}

While countries in SSA each face a unique set of circumstances, all told these challenges are not terribly dissimilar from Nepal's situation 20 years ago. Remote population distribution with underdeveloped roads and infrastructure, meager government funding, and a paucity of well-trained surgeons echo the barriers faced by Nepal 20 years ago. These challenges hinder the provision of high-quality care, 
and low-quality services suppress demand and perpetuate the problem. ${ }^{17}$ Self-sustaining eye-care systems must be rooted in quality services, which are necessary to generate sufficient demand, drive innovation, strengthen personnel, and ultimately attract investment in eye care by health ministries. As Ruit is noted for saying, "Even the poorest of the poor understand quality."

Quality in Ethiopia and Ghana is rising. Intraocular lenses with biometry for CS, once the exception, are now commonplace and universal among HCP partners. Patient surgery and outcome data are recorded and analyzed (compared to WHO guidelines) to iterate and improve efforts. Fellowshiptrained retina specialists, pediatric ophthalmologists, cornea surgeons, and glaucoma specialists operate within each country, and young, promising ophthalmologists are entering prestigious fellowship training programs in India, South Africa, Canada, and Nepal. Continued support and in-country training allows them to face challenges as they arise, rather than falling into the category of underutilized, highly trained specialists.

Training standards, explicitly defined and rigorously maintained, will continue to drive the rise in quality observed in these African countries. For this reason, emphasis is placed on residency education and surgical training. Initiatives include coordination of a committee of residency directors in Ethiopia, which has better aligned incentives by disarticulating the residency directorship from competing administrative roles. Grants obtained through the American Society of Cataract and Refractive Surgery fund annual training meetings for residency directors, a profound manifestation of the "train the trainer" mantra that permeated Ruit's work in Nepal. Plans to offer fellowships in subspecialties are well under way in both Ghana and Ethiopia. High-quality care stands as a pillar of the compassionate-capitalism model, driving demand among paying patients who then fund charitable care for the poor and sustain project growth. As quality standards improve, awareness grows, patients seek surgery sooner and the market created by an emerging middle class can be captured.

As previously mentioned, in the USA, where CS quality is extremely high, the majority of cataract patients undergo surgery with vision that would not even qualify as impaired by WHO standards. This is the same standard of care that should be pursued in emerging economies. Indeed, we have seen patients seeking CS sooner and even having elective surgery in Nepal, where quality remains high. The same trend can be observed in its early stages in areas of SSA; however, data remain sparse and insufficient evaluation and monitoring has been conducted to assess progress.

\section{Revenue streams and reappropriation}

Both Ghana and Ethiopia have centralized healthcare systems. In Ethiopia, there is little to no government support for eye-care services. In Ghana, the National Health Insurance Scheme covers eye-care procedures, such as CS for adults; however, payouts to the provider (public or private) are notoriously late, though they do arrive. Many eye-care services are inadequately reimbursed or not reimbursed at all, such as surgeries for children. The financial remuneration for doctors and nurses in Africa is quite low due to these and other barriers. There are particularly low government salaries and poor incentives to increase productivity. In this environment of poor incentives, quality does not improve, and without high-quality driving demand, volume will remain low. Similarly, lower volume decelerates the training of new surgeons, again lowering overall quality. Consequently, many well-intentioned and talented ophthalmologists leave government jobs for higher-paying work at foreign-owned, for-profit hospitals. The infrastructure does not currently exist to incentivize high-quality, high-volume care in a system that can provide free or subsidized care with profit. These realities are distinct from the healthcare landscape encountered in Nepal. The reimbursement models in Ghana and Ethiopia present challenges to the compassionate-capitalism model that so successfully drove sustainability and growth in Nepal, as well as in India.

The Aravind Eye Hospital and TIO are both private institutions, dedicated to ophthalmic care and unencumbered by external financial interests and operation. Although both institutions have worked successfully with the government in implementing broad eye-care initiatives, institutional finances are self-governed. Reappropriating revenue to fund new eyecare projects or charitable care are decisions made internally.

HCP's primary partners in Africa - eye centers and ophthalmology departments that are entities within the context of a government teaching hospital - face difficulties in ensuring profits are reinvested into their own departments, much less into charitable or free care. Negotiating incentive-based agreements with both the teaching hospitals and the health ministries has been an important objective unique to these countries. Advocacy and well-maintained relationships with hospital administrators and ministry officials are paramount in ensuring eye-care revenue is not siphoned to larger or more politically powerful departments.

Participating in and encouraging the development of national eye-care plans alongside health ministries and governing bodies and according to the strategies outlined 
by the WHO's global action plan ${ }^{4}$ engenders buy-in and aligns prominent stakeholders. Innovative concepts, such as a national outreach committee in Ghana, can adapt the compassionate capitalism model within a centralized healthcare system. Though the public sector traditionally lacks the nimble flexibility the private and NGO sectors allow, an established national insurance scheme and centralized governance offer a framework for reimbursement and the possibility of coordinated action on which a sustainable solution can be built. The question of whether such a solution will manifest remains unanswered.

\section{Opportunity in primary eye care}

Primary eye health is a significant public health consideration that also presents an opportunity for addressing some of the economic challenges of eye care in Africa. Uncorrected refractive error and cataract together cause $>70 \%$ of moderate and severe visual impairment, including blindness $(<3 / 60){ }^{2}$ The economic detriment of such visual impairment is well documented and reverberates throughout a society. A blind member of a community takes on average 2.5 people from the labor force to provide adequate care. Often, this means children who are kept home from school and robbed of an education, thereby deepening the cycle of poverty within a family. In the USA alone, an estimated US\$49 billion is lost each year due to diminished productivity caused by blindness. ${ }^{35}$ However, the return on investment directed into eye-care development is profound: estimated at $400 \%$, or $\$ 4$ to every $\$ 1$ invested, in a recent PricewaterhouseCoopers study. ${ }^{23}$ The broad economic gain of establishing effective systems of eye-care rests conservatively at US\$100-500 billion. $^{23}$

Treating visual impairment, including uncorrected refractive error, opens job opportunities and enhances personal safety and productivity. Studies have demonstrated that the visually impaired are three times as likely to be involved in a motor vehicle accident and two times as likely to suffer groundlevel falls. Those with corrected vision find employment at a rate three times those who are visually impaired. ${ }^{3}$ Even more sobering, a 2016 study published in the British Journal of Ophthalmology showed blind people in the developing world have a mortality rate $33 \%$ greater than their age-matched peers. ${ }^{36}$ One of the key challenges will be conveying the problem as well as the possibility to policy makers and thereby elevating the prioritization of eye care in health expenditure.

Effective models that can scale up and meet the needs of the African landscape must include the establishment or expansion of a country's primary/community eye-care systems through infrastructure development, technical assistance, and training.
Training nurses and community health workers who can integrate primary eye care and refractive services into their scope of work is of particular importance, including vitamin A distribution, basic diagnostics, referral, and provision of glasses. Telemedicine and mobile technologies, such as smartphoneenabled ophthalmic imaging, are empowering community health workers - whether ophthalmic technicians, nurses, or primary physicians - to integrate primary eye-care services with public healthcare initiatives, such as diabetes-screening campaigns. They also pave the way for increased efficiency of utilization of very scarce personnel - ophthalmologists.

An emerging, innovative area of refractive care that can facilitate the compassionate capitalism model of recycled revenue generation is refractive surgery or "laser vision correction.” In Nepal, HCP and TIO developed a refractive surgery center, pioneering the concept in the developing world. The refractive surgery center not only serves as a profit generator but also offers compassionate, charitable care for those with refractive error. The viability of refractive surgery in low-resource settings has now been proven in Nepal, India, and China. Plans for refractive surgery centers in Ethiopia and Ghana are under way, with the expectation that (similar to Nepal) they will fuel growth and broaden the eye-care ecosystem as profits are reinvested into charitable care and project expansion. Early market analysis and financial projections predict that a refractive surgery center can be profitable in these markets within 3 years of operation.

In a more centralized political and economic environment, where eye-care revenue is often subsumed by higher-priority programs, creative mechanisms for revenue generation and appropriation are necessary. Partnering with private hospitals and private industry to develop refractive services, whether optical or surgical, provide avenues toward financial sustainability. However, without deeper commitment to eye care by the public sector and profound elevation of surgical quality across the landscape, the promise of financially sustainable eye-care programs remains elusive.

\section{Conclusion}

The problem of avoidable blindness in Africa has not been solved. Viable solutions from Nepal and India offer promise, but remain in their adolescence, needing additional funding and facing challenges unique to the African landscape. With the highest prevalence of avoidable blindness and the highest projected population growth, SSA represents an enormous challenge for the global community. The magnitude of the problem will only grow as the population continues to multiply and age. The entanglement of poverty and blindness 
is inexorable and undeniable, pressing for a sustainable solution that addresses the immense shortage of trained eye-care providers. Keys for sustainability include use of evidencebased approaches, the use of robust mechanisms to monitor and evaluate programs, development of the compassionate capitalism mode ${ }^{11}$ to foster financial independence, commitments from health ministries to dedicate more resources to eye health, and association with related developmental initiatives (eg, those affecting such areas as poverty alleviation).

The transformation of Nepal's eye-care system, which effectively reversed its rate of blindness, provides a model that meets these key measures and is beginning to replicate in places such as Ethiopia and Ghana. Though key questions remain unanswered, evidence to date demonstrates this model can proliferate sustainably in Africa, though additional funding and resource commitments are needed. Government prioritization and deeper financial support from the philanthropic community will ultimately determine if success is actualized and then sustained.

\section{Disclosure}

The authors report no conflicts of interest in this work.

\section{References}

1. Bourne RR, Flaxman SR, Braithwaite T, et al. Global causes of blindness and distance vision impairment 1990-2020: a systematic review and meta-analysis. Lancet Glob Health. 2017;5(12):e1221-e1234.

2. Bourne RR, Flaxman SR, Braithwaite T, et al. Magnitude, temporal trends, and projections of the global prevalence of blindness and distance and near vision impairment: a systematic review and meta-analysis. Lancet Glob Health. 2017;5(9):e888-e897.

3. World Health Organization. Universal Eye Health: A Global Action Plan 2014-2019. Geneva: WHO; 2013.

4. Vision 2020 Australia. Universal eye health: a global action plan 2014-2019. Available from: http://www.vision2020australia.org. au/resources/universal-eye-health-a-global-action-plan-2014-2019. Accessed April 17, 2018.

5. Lindstrom R. Thoughts on cataract surgery: 2015. 2015. Available from: https://www.reviewofophthalmology.com/article/thoughts-on--cataractsurgery-2015. Accessed October 19, 2017.

6. Brilliant LB, Pokhrel RP, Grasset NC, et al. Epidemiology of blindness in Nepal. Bull World Health Organ. 1985;63(2):375-386.

7. Nepal Netra Jyoti Sangh. The epidemiology of blindness in Nepal 2012. 2012. Available from: https://www.iapb.org/wp-content/uploads/ Epidemiology-of-Blindness-Nepal.pdf. Accessed October 19, 2017.

8. Ruit S, Paudyal G, Gurung R, Tabin G, Moran D, Brian G. An innovation in developing world cataract surgery: sutureless extracapsular cataract extraction with intraocular lens implantation. Clin Exp Ophthalmol. 2000;28(4):274-279.

9. Gogate PM, Kulkarni SR, Krishnaian S, et al. Safety and efficacy of phacoemulsification compared with manual small-incision cataract surgery by a randomized controlled clinical trial: six-week results. Ophthalmology. 2005;112(5):869-874.

10. Ruit S, Tabin G, Chang D, et al. A prospective randomized clinical trial of phacoemulsification vs manual sutureless small-incision extracapsular cataract surgery in Nepal. Am J Ophthalmol. 2007;143(1):32-38.

11. Tabin $\mathrm{G}$. The cataract blindness challenge innovations case discussion: Aravind Eye Care System. Innov Technol Gov Glob. 2007;2(4):53-57.
12. Bhandari A, Dratler S, Raube K, Thulasiraj RD. Specialty care systems: a pioneering vision for global health. Health Aff (Millwood). 2008;27(4):964-976.

13. International Agency for the Prevention of Blindness. Cataract surgery protocol - Nepal. 2015. Available from: https://www.iapb.org/resources/ cataract-surgery-protocol-nepal. Accessed October 19, 2017.

14. United Nations. World Population Prospects - The 2017 Revision: Key Findings and Advance Tables. New York: UN; 2017.

15. Frick KD, Foster A, Bah M, Faal H. Analysis of costs and benefits of the Gambian Eye Care Program. Arch Ophthalmol. 2005;123(2):239-243.

16. Naidoo K, Gichuhi S, Basáñez MG, et al. Prevalence and causes of vision loss in sub-Saharan Africa: 1990-2010. Br J Ophthalmol. 2014;98(5):612-618.

17. Gogate P, Kalua K, Courtright P. Blindness in childhood in developing countries: time for a reassessment? PLoS Med. 2009;6(12):e1000177.

18. Singh A, Strauss GH. High-fidelity cataract surgery simulation and third world blindness. Surg Innov. 2015;22(2):189-193.

19. Akin JS, Guilkey DK, Denton EH. Quality of services and demand for health care in Nigeria: a multinomial probit estimation. Soc Sci Med. 1995;40(11):1527-1537.

20. Palmer J, Chinanayi F, Gilbert A, et al. Mapping human resources for eye health in 21 countries of sub-Saharan Africa: current progress towards Vision 2020. Hum Resour Health. 2014;12:44.

21. Habtamu E, Eshete Z, Burton MJ. Cataract surgery in southern Ethiopia: distribution, rates and determinants of service provision. BMC Health Serv Res. 2013;13:480.

22. Habtamu E, Rajak SN, Gebre T, et al. Clearing the backlog: trichiasis surgeon retention and productivity in northern Ethiopia. PLoS Negl Trop Dis. 2011;5(4):e1014.

23. Fred Hollows Foundation. Investing in vision: a study by PricewaterhouseCoopers. 2017. Available from: http://www.hollows.org/au/ research/investing-in-vision. Accessed October 19, 2017.

24. du Toit R, Faal HB, Etya'ale D, et al. Evidence for integrating eye health into primary health care in Africa: a health systems strengthening approach. BMC Health Serv Res. 2013;13:102.

25. Lewallen S, Thulasiraj RD. Eliminating cataract blindness: how do we apply lessons from Asia to sub-Saharan Africa? Glob Public Health. 2010;5(6):639-648.

26. Aravind Eye Care System. Aravind news. 2014. Available from: http:// www.aravind.org/default/aravindnewscontent/NI00000134. Accessed March 10, 2018.

27. Magrabi ICO Cameroon Eye Institute. Africa Eye Foundation establishes the Magrabi ICO Cameroon Eye Institute. 2018. Available from: https://www.micei.org/about. Accessed March 10, 2018.

28. Seva Foundation. Kilimanjaro Centre for Community Ophthalmology. Available from: http://www.seva.org/site/PageNavigator/programs/ partners/KCCO. Accessed March 10, 2018.

29. Dr Agarwal's Eye Hospital. Africa branches. 2016. Available from: http://www.dragarwal.com/africa-branches. Accessed March 10, 2018.

30. IABP [homepage]. Available from: www.iabp.org. Accessed October $19,2017$.

31. Mathenge W, Nkurikiye J, Limburg H, Kuper H. Rapid assessment of avoidable blindness in Western Rwanda: blindness in a postconflict setting. PLoS Medicine. 2007;4(7):e217.

32. World Health Organization. Global Initiative for the Elimination of Avoidable Blindness: Action Plan 2006-2011. Geneva: WHO; 2007.

33. Witter S, Garshong B. Something old or something new? Social health insurance in Ghana. BMC Int Health Hum Rights. 2009;9:20.

34. Health Systems 20/20 Project. An Evaluation of the Effects of the National Health Insurance Scheme in Ghana. Washington, DC: US Agency for International Development; 2009.

35. The economic burden of vision loss and eye disorders in the United States. Available from: https://www.preventblindness.org/sites/default/ files/national/documents/Impact_of_Vision_Problems.pdf. Accessed October 19, 2017.

36. Thomas BJ, Sanders DS, Oliva MS, et al. Blindness, cataract surgery and mortality in Ethiopia. Br J Ophthalmol. 2016;100(9):1157-1162. 
Innovation and Entrepreneurship in Health is an international, peer reviewed, open access journal publishing original research, reports, reviews and commentaries on innovation and entrepreneurship in health. Special focus will be given to the theory, process, and practice of innovation and entrepreneurship by individuals and organizations within the health care context globally. The manuscript management system is completely online and includes a very quick and fair peer review system, which is all easy to use. Visit http://www.dovepress.com/ testimonials.php to read real quotes from published authors. 